Canadian Journal of Applied Linguistics

Revue canadienne de linguistique appliquée

\title{
Les homophones grammaticaux, portrait actuel des occurrences et des taux de réussite chez des élèves de 9 à 12 ans
}

\section{Marie-Hélène Giguère and Rebeca Aldama}

Volume 22, Number 2, 2019

URI: https://id.erudit.org/iderudit/1067311ar

DOI: https://doi.org/10.7202/1067311ar

See table of contents

Publisher(s)

University of New Brunswick

ISSN

1920-1818 (digital)

Explore this journal

Cite this article

Giguère, M.-H. \& Aldama, R. (2019). Les homophones grammaticaux, portrait actuel des occurrences et des taux de réussite chez des élèves de 9 à 12 ans. Canadian Journal of Applied Linguistics / Revue canadienne de linguistique appliquée, 22(2), 133-155. https://doi.org/10.7202/1067311ar

\section{Article abstract}

This study aims to describe occurrences of homophone words and frequencies of grammatical homophone errors in 119 free texts of 9 to 12-year-old Quebec students from a list of 22 series of homophones. This analysis made it possible to confirm that grammatical homophones are widely used by students, but that, considering all the possibilities in the different series, they are more successful than we had anticipated. Thus, our results made it possible not only to establish a current portrait of the homophone words spontaneously used by 9 to 12-year-old students in their texts, but also to identify constants in the successes (related to the frequency). Some syntactic contexts were also analyzed to identify priority areas to work on for teachers. Finally, our study identifies areas of vulnerability among students based on three factors: gender, age, and socio-economic background. The discussion concludes with recommendations for teaching grammatical categories.
Copyright (c) Marie-Hélène Giguère, Rebeca Aldama, 2020

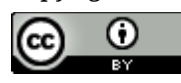

This document is protected by copyright law. Use of the services of Érudit (including reproduction) is subject to its terms and conditions, which can be viewed online.

https://apropos.erudit.org/en/users/policy-on-use/ 


\title{
Les homophones grammaticaux, portrait actuel des occurrences et des taux de réussite chez des élèves de 9 à 12 ans
}

\author{
Marie-Hélène Giguère \\ Université du Québec à Montréal \\ Rebeca Aldama \\ Université du Québec à Montréal
}

\begin{abstract}
Résumé
La présente étude vise à décrire des occurrences des mots de forme homophone et des fréquences d'erreurs d'homophones grammaticaux dans 119 textes libres d'élèves québécois de 9 à 12 ans à partir d'une liste de 22 séries d'homophones. Cette analyse a permis de confirmer que les homophones grammaticaux sont très utilisés par les élèves, mais qu'en considérant toutes les possibilités dans les différentes séries, ces derniers sont mieux réussis que ce que nous avions anticipé. Ainsi, nos résultats ont non seulement permis d'établir un portrait actuel des mots homophones utilisés spontanément par des élèves de 9 à 12 ans dans leurs textes, mais également d'établir des constantes dans les réussites (liées à la fréquence). Certains contextes syntaxiques ont également été analysés pour permettre d'établir les zones prioritaires à travailler pour les enseignants. Enfin, notre étude permet d'établir des zones de vulnérabilité chez les élèves en fonction de trois facteurs : le sexe, l'âge et le milieu socioéconomique. La discussion conclut sur des recommandations pour l'enseignement des catégories grammaticales.
\end{abstract}

\begin{abstract}
This study aims to describe occurrences of homophone words and frequencies of grammatical homophone errors in 119 free texts of 9 to 12-year-old Quebec students from a list of 22 series of homophones. This analysis made it possible to confirm that grammatical homophones are widely used by students, but that, considering all the possibilities in the different series, they are more successful than we had anticipated. Thus, our results made it possible not only to establish a current portrait of the homophone words spontaneously used by 9 to 12-year-old students in their texts, but also to identify constants in the successes (related to the frequency). Some syntactic contexts were also analyzed to identify priority areas to work on for teachers. Finally, our study identifies areas of vulnerability among students based on three factors: gender, age, and socio-economic background. The discussion concludes with recommendations for teaching grammatical categories.
\end{abstract}




\section{Les homophones grammaticaux, portrait actuel des occurrences et des taux de réussite chez des élèves de 9 à 12 ans}

La langue française, comme l'a démontré Catach (1995), possède une orthographe semi-transparente qui peut s'expliquer par un plurisystème. En effet, des graphèmes transposent des sons de la langue (phonogrammes), certains expriment du sens (les morphogrammes) et d'autres permettent de distinguer visuellement des mots homophones (logogrammes). Les homophones sont définis par McNicoll, Roy et Université de Sherbrooke (1984) comme « des termes de sens différents, identiques par la prononciation, mais semblables ou non par la forme écrite » (p. 14).

Dans le but d'aider les élèves à s'approprier des connaissances pour surmonter cette difficulté liée à la langue écrite, les programmes de formation québécois ont mis à l'étude cette notion en 1979, tant au primaire qu' au secondaire. Il est ainsi possible de trouver des listes de mots ${ }^{1}$ homophones classés par paires (ou séries) qui devaient faire l'objet d'enseignement et d'évaluation à certaines années précises dans la scolarité (Ministère de l'Éducation du Québec [MEQ], 1979). Or, depuis 2000, les programmes de formation ont complètement retiré cette notion de l'enseignement (MEQ, 2001). Actuellement, les documents officiels comme la Progression des apprentissages en français (2009 au primaire et 2011 au secondaire) insistent davantage sur les raisonnements grammaticaux complets qui permettent à l'élève de distinguer les classes de mots en contexte ( $\mathrm{p}$. ex. travaille et travail, Ministère de l'Éducation, du Loisir et du Sport [MELS], 2009, 2011).

Pourquoi s'intéresser aux homophones alors qu'ils ne font plus partie du programme de formation québécois depuis 2000 ? D'une part, cette notion attire encore l'attention de chercheurs en éducation parce que les erreurs liées aux homophones font partie des erreurs les plus fréquentes, voire persistantes, chez les jeunes scripteurs (Boivin et Pinsonneault, 2012, 2018 ; Brissaud et Cogis, 2011 ; Champoux, 2015 ; Jaffré, 2006). D'autre part, bien qu'aucune recherche récente ne porte spécifiquement sur les pratiques des enseignants au sujet des homophones, notre expérience en salle de classe, nos observations en contexte de recherche ainsi que l'examen de manuels scolaires, de cahiers d'exercices et de feuilles d'activités téléchargeables nous renseignent sur le fait que l'enseignement des homophones se maintient dans les pratiques des enseignants. Cette situation est également relevée par Champoux (2015) et par Tallet (2016a). En effet, il semble que leur enseignement fait partie d'une culture scolaire, un legs d'anciens programmes, qui se manifeste sous la forme d'habitude dans les pratiques chez beaucoup d'enseignants sans faire l'objet de remise en question (Tallet, 2016a). Au fil des ans, les élèves continuent d'apprendre les homophones surtout par le biais de longues listes de paires ou de séries à distinguer bien souvent par des « trucs », soit des remplacements, par exemple de remplacer une finale en /E/ par mordre ou mordu (Boivin et Pinsonneault, 2012 ; Champoux, 2015 ; Fisher, 1994 ; Nadeau et Fisher, 2006 ; Tallet, 2016b). La mémorisation de ces listes et de ces « trucs » fait non seulement courir le risque de nombreuses confusions (Nadeau et Fisher, 2006 ; Tallet, 2016b), mais s'accompagne également d'exercices de phrases trouées et de dictées évaluatives qui ne soutiennent pas la construction de savoirs transférables chez les élèves (Duret et Zecca, 2014 ; Nadeau et Fisher, 2006). Cette culture et le matériel disponible inciteraient les enseignants à faire perdurer cet enseignement. Pourtant, puisque les listes d'homophones ne font pas partie des programmes, le choix des séries repose sur les préférences des enseignants, probablement influencés par le matériel didactique mis à leur disposition. 
Nous avons d'ailleurs recensé plusieurs cahiers d'exercices québécois publiés après la Progression des apprentissages (MELS, 2009) qui présentent ce type d'exercices au $3^{\mathrm{e}}$ cycle du primaire, sans compter le matériel disponible en ligne ou celui développé dans les écoles. Le matériel didactique offert n'est donc pas en accord avec les directives ministérielles, mais conforte les enseignants dans leurs pratiques d'enseignement des homophones. De plus, ceci représente une contradiction avec les résultats d'études révélant que cet enseignement crée justement des confusions dans l'apprentissage de la langue écrite (Fisher, 1994 ; Nadeau et Fisher, 2006 ; Tallet, 2016a).

Ainsi, les erreurs liées aux mots homophones perdurent chez les jeunes scripteurs, tout comme les pratiques d'enseignement inadéquates et pourtant découragées par les chercheurs et les programmes officiels. Les années et les programmes de formation n'ont pas suffi à faire évoluer l'enseignement de l'orthographe des mots de forme homophone, ni à faire diminuer les erreurs des élèves dans leurs textes (Boivin et Pinsonneault, 2014, 2018 ; Champoux, 2015).

À ces problèmes s'ajoute celui de la méthode souvent utilisée pour documenter les difficultés des élèves. En effet, les recherches portant sur la réussite des mots homophones se basent souvent sur des outils méthodologiques comme des dictées ou des textes à corriger dans lesquelles la syntaxe, la grammaire et le choix du lexique visent à explorer plusieurs contextes grammaticaux (Champoux, 2015 ; McNicoll et coll., 1984 ; Tallet, 2016b). Par exemple, pour savoir si l'élève discrimine les formes concurrentes pour orthographier sur/sûr/sure et s'il est en mesure d'orthographier correctement en contexte, plusieurs phrases lui sont proposées dans lesquelles toutes les graphies sont nécessaires. Il serait intéressant de connaitre aussi le choix de mots que font les élèves dans un contexte de production écrite d'un texte portant sur un sujet libre. Dans des contextes syntaxiques qui sont les leurs, le choix des mots, incluant les mots homophones, pourrait être influencé par leur capacité à bien y réfléchir et donc à bien les orthographier.

Ainsi, cet article vise à présenter les résultats d'une recherche ${ }^{2}$ décrivant l'utilisation d'homophones grammaticaux par des élèves de 9 à 12 ans dans des textes libres. Trois questions de recherche nous ont guidées : 1. Quels mots homophones utilisent-ils réellement ? 2. Est-ce que certains homophones méritent vraiment un enseignement plus rigoureux en raison des difficultés qu'ils posent effectivement aux élèves lors d'une activité d'écriture de texte ? 3. Est-ce que les taux de réussite étaient associés au milieu socioéconomique des élèves, à leur âge et à leur sexe comme l'ont suggéré Desrosiers et Tétrault (2012) à propos des textes ? Quelques études ont montré l'influence du sexe et du statut socioéconomique des élèves pour expliquer les écarts entre les taux de réussite aux épreuves obligatoires (Boivin et Pinsonneault, 2018 ; Desrosiers et Tétrault, 2012). Cette recherche veut observer les effets de ces variables sur l'utilisation et la réussite des mots homophones en situation d'écriture libre.

La section suivante s'attarde à définir le concept d'homophonie et à présenter les résultats de différentes recherches portant sur les occurrences des mots homophones dans les écrits d'élèves québécois de 9 à 12 ans, puisqu'il s'agit de la population étudiée dans ce corpus. La méthodologie sera ensuite présentée, suivie des résultats et d'une discussion qui permettra de répondre aux questions de recherche. 


\section{Cadre conceptuel}

Cette section a comme objectif de décrire le concept d'homophone. Elle traite ensuite des approches pédagogiques pour leur enseignement et se termine par les portraits recensés dans la littérature scientifique à propos des occurrences et réussites chez des élèves québécois.

\section{Qu'est-ce qu'un homophone ?}

Un des grands défis des élèves francophones en écriture se rapporte à l'opacité de l'orthographe (Catach, 1995 ; Goswami, Gombert et Barrera, 1998 ; Mousty et Alegria, 1999). En français, la relation entre les unités sonores à l'oral et à l'écrit n'est pas régulière ni systématique : plusieurs mots fréquents se prononcent de la même manière, mais s'écrivent de diverses façons (Jaffré, 2006 ; McNicoll et Roy, 1985). Pour faciliter la lecture, la forme écrite des mots prononcés de la même manière à l'oral se distingue à l'écrit. Ainsi, en voyant un mot écrit d'une telle façon, le lecteur lui attribue un seul sens (McNicoll et Roy, 1985). En revanche, la difficulté survient à l'écrit puisque le scripteur doit opter pour la graphie correcte à la suite de l'analyse syntaxique du contexte du mot, surtout en ce qui concerne les homophones grammaticaux.

Dans les anciens programmes de formation (MEQ, 1979), on nommait « homonymes » cette réalité linguistique (homo $=$ semblable $;$ onyme $=$ mot $)$, désignée aujourd'hui comme « homophones » $($ homo $=$ semblable ; phone $=$ son $)$. Cette précision du métalangage vise à circonscrire le concept autour des mots de sens différents, mais de prononciation semblable. ${ }^{3}$ Il est alors possible d'exclure les mots qui s'écrivent de la même manière, mais qui n'ont pas la même prononciation qui sont des homonymes, mais pas des homophones (p. ex. aller vers l'est; il est ici). De plus, selon la Banque de Dépannage Linguistique (BDL) :

On distingue les homophones lexicaux et les homophones grammaticaux. On parle d'homophones lexicaux lorsque la ressemblance existe entre des mots lexicaux, c'est-à-dire les noms, les verbes, les adjectifs et les adverbes. Il y a homophonie lexicale entre des mots qui appartiennent habituellement à la même catégorie grammaticale. On parle d'homophones grammaticaux lorsque la ressemblance existe entre des mots grammaticaux, c'est-à-dire les déterminants, les pronoms, les prépositions et les conjonctions. (Office de la langue française du Québec, 2018, «Qu'est-ce qu'un homophone lexical ?», s.p.)

Aussi, selon Fisher (1994), les homophones grammaticaux correspondent généralement aux mots homophones qui appartiennent à des catégories grammaticales différentes (p. ex. $\grave{a}-a$, on-ont, $(e-s e)$. Ce sont ces derniers qui ont retenu notre attention pour cette recherche puisqu'ils sont les plus abordés dans le matériel scolaire et les plus enseignés par des « trucs » aux élèves, orientant ainsi leurs décisions grammaticales sans nécessairement qu'un raisonnement complet n'ait lieu sur les séries (Champoux, 2015 ; Fisher, 1994; Nadeau et Fisher, 2006).

Tallet (2016b) a qualifié les homophones d'ensemble « fourre-tout » de couples ou de séries de mots monosyllabiques grammaticaux et lexicaux d'une très haute fréquence. Comme ils ne partagent que des traits sonores, ils ne forment pas une catégorie du point de 
vue grammatical ; ils ne sont pas une classe de mots. Selon cette chercheuse, il s'agit « d'un ensemble inventé par l'école dans le but d'aider les élèves à les orthographier correctement. » (Tallet, 2016b, p. 3). Brissaud et Cogis (2011) ont ajouté : « l'idée [des « trucs »] est que l'orthographe des homophones est trop difficile à analyser et qu'il vaut mieux doter les élèves d'un "truc" efficace pour éviter les fautes» (p. 263). Ces « trucs», comme par exemple le remplacement de on par Léon, issus des anciens programmes de formation, viseraient à « réduire l'apprentissage de la terminologie grammaticale et la connaissance explicite des règles » (Nadeau et Fisher, 2006, p. 60), ce qui est contraire à ce que nous savons aujourd'hui sur la réussite en orthographe (Nadeau et Fisher, 2011, 2014). Il est donc possible de penser que ces «trucs » aient surtout été proposés comme support pour aider les élèves, particulièrement les élèves en difficulté et à risque d'échouer.

\section{Approches d'enseignement}

Pourtant, cette approche par «trucs » ne serait pas à prioriser selon Boivin et Pinsonneault (2012), car elle risque d'amener les élèves à traiter les homophones comme une catégorie grammaticale à part entière. En outre, elle ne leur permettrait pas de développer une compréhension juste du système linguistique (Fisher, 1994). Il est ainsi recommandé de développer la connaissance des catégories grammaticales et des structures syntaxiques pour favoriser la compréhension et la maîtrise du code écrit (Boivin et Pinsonneault, 2012, 2018 ; Champoux, 2015). Plusieurs chercheurs ont proposé d'entraîner les élèves à raisonner et à réfléchir sur la langue (Brissaud et Cogis, 2011 ; Cogis, 2005 ; Nadeau et Fisher, 2006) et à mettre en place des exercices qui offrent des situations authentiques d'écriture, pour ainsi favoriser le transfert des connaissances (Cogis et Brissaud, 2003).

En abordant la question de l'enseignement, il devient alors nécessaire de reconnaître que la fréquence des homophones dans la langue et l'analyse syntaxique qu'ils nécessitent rend difficile le choix de l'orthographe pour les scripteurs à propos d'un bon nombre d'homophones à chacune des phrases dans laquelle ils se trouvent (McNicoll et coll., 1984). En effet, selon Catach (1995), les mots de forme homophone constituent $6 \%$ des mots de la langue. Il existe, bien sûr, des logiciels de fréquence lexicale, comme Manulex, qui proposent des listes de fréquences de mots. Or, nous désirons contribuer, par cette étude, à établir une fréquence des mots de forme homophone provenant des écrits libres des élèves plutôt qu'à partir de manuels scolaires (comme Manulex), ce qui en constitue son originalité.

La prochaine section permettra d'étudier les occurrences des homophones en lien avec les erreurs observées chez de jeunes scripteurs au Québec.

\section{Les occurrences et les erreurs dans les textes d'élèves québécois}

Des recherches récentes (Boivin et Pinsonneault, 2012, 2014, 2018 ; Champoux, 2015 ; Duret et Zecca, 2014 ; Tallet, 2016a, 2016b) ont remis la problématique des mots homophones à l'avant-scène. En effet, des études sont davantage publiées sur le sujet, tant en France qu'au Québec, pour décrire à la fois les erreurs des élèves, mais surtout les stratégies d'enseignement qui sont à réviser et à améliorer. 
En ce qui a trait à la description des erreurs des élèves du primaire dans des corpus de textes, il faut remonter quelques décennies dans le temps pour repérer l'étude de McNicoll et coll. en 1984, au moment où le programme de français au Québec comportait 19 paires d'homophones à l'étude (MEQ, 1979). Ces auteurs ont réalisé une recension des erreurs dans les textes de 48 élèves répartis dans des classes de $3^{\mathrm{e}}, 4^{\mathrm{e}}$ et $6^{\mathrm{e}}$ année d'une école primaire au Québec. Leurs résultats ont montré les fréquences de 22 paires (ou séries) homophoniques utilisées par les élèves et ont identifié des zones de vulnérabilité, à savoir les formes disjointes (p. ex. $\left.t^{\prime} a, m^{\prime} a, l^{\prime} a, m^{\prime} o n t\right)$ et les paires pronom/verbe (on/ont). Leurs résultats ont montré, d'autre part, que les formes simples et fréquentes étaient mieux réussies par les élèves que les formes plus rares et disjointes (p. ex. $\mathrm{ma} / \mathrm{m}$ 'a). Certaines catégories grammaticales, comme les déterminants, étaient également mieux réussies par les élèves.

Ce n'est que tout récemment, avec les études de Boivin et Pinsonneault (2012, 2018) et Champoux (2015) qu'on a obtenu un portrait plus actuel des erreurs commises par 136 élèves du secondaire. Les finales en $/ E$ / semblent les plus problématiques, suivies par la série des ses/ces/c'est/s'est et des paires à/a et ce/se (Champoux, 2015). Selon Boivin et Pinsonneault $(2012,2018)$, les erreurs liées aux homophones grammaticaux constituent $15 \%$ des erreurs qu'elles analysent comme appartenant à la syntaxe dans leur corpus. En effet, il est possible d'analyser ce type d'erreur comme entrainant des phrases asyntaxiques si, par exemple, les constituants essentiels de la phrase sont erronés (dans la phrase * Ont à des problèmes, il n'y a pas de sujet ni de verbe au sens littéral). D'autre part, un rapport de recherche publié en 2014 par ces mêmes auteures, effectué à partir de 969 textes issus des épreuves ministérielles de fin de cycle en écriture, a montré que les erreurs liées aux homophones représentaient une moyenne de 2,86 erreurs sur 100 mots en $4^{\mathrm{e}}$ année du primaire avec un écart-type de 1,94 et diminuaient à 1,33 erreur sur 100 mots en moyenne, avec un écart-type de 1,50 , en $6^{\mathrm{e}}$ année. Certains élèves de $4^{\mathrm{e}}$ année ont fait jusqu'à 10,07 erreurs sur 100 mots contre 8,24 pour les élèves de $6^{\mathrm{e}}$ année. Les résultats ont montré que le taux d'erreurs diminuait significativement entre la $4^{\mathrm{e}}$ année du primaire et la $5^{\mathrm{e}}$ année du secondaire où le nombre moyen d'erreurs était de 0,43 pour un maximum d'erreur de 3,23 sur 100 mots (le minimum atteint 0 ).

Enfin, il a été démontré par Brissaud, Chevrot et Lefrançois (2006) que le choix de la graphie des formes verbales en $/ E$ / revêtait un caractère développemental entre 8 et 15 ans. L'âge des élèves avait donc une incidence sur le choix de certaines graphies en $/ E /$ : en $3^{\mathrm{e}}$ année, la forme $-e r$ était plus disponible que la forme $-e ́$. Cette tendance s'est inversée en $4^{\mathrm{e}}$ année : la forme $-e ́$ est devenue plus disponible que $-e r$. Il est à noter que la Progression des apprentissages en français (MELS, 2009) proposait l'enseignement des temps composés à partir du $3^{\mathrm{e}}$ cycle du primaire, mais que les élèves devaient identifier un infinitif à partir de la $3^{\mathrm{e}}$ année.

Ces dernières études ne recensaient cependant pas les occurrences des mots homophones dans les textes, mais uniquement les erreurs. Autrement dit, elles ne s'attardaient qu'aux formes erronées sur un nombre total de mots, mais jamais au nombre d'occurrences réussies ou au taux de réussite. Ainsi, à la suite de ces recensions, il nous est apparu pertinent, à la fois pour les didacticiens et pour les praticiens, de cibler trois objectifs pour répondre à nos trois questions de recherche: d'abord, (a) décrire les occurrences des différents homophones grammaticaux utilisés par des élèves de 9 à 12 ans de même que (b) leurs réussites et leurs erreurs. Ensuite, il nous est apparu indiqué de savoir (c) si leur milieu socioéconomique, leur âge et leur sexe ont un impact sur les taux de 
réussite non pas seulement en écriture de textes comme l'ont suggéré certaines études (Desrosiers et Tétrault, 2012), mais également sur cet aspect précis de la langue. Ce faisant, le portrait établi permettra d'observer si le portrait dégagé par l'étude de McNicoll et Roy en 1985 a évolué.

\section{Méthodologie}

Tel que mentionné dans la problématique, cette recherche visait à décrire l'utilisation d'homophones grammaticaux en focalisant sur des occurrences d'homophones dans des écrits d'élèves de 9 à 12 ans. Elle visait à décrire également le taux de réussite pour chaque série d'homophones dans un contexte où l'élève choisissait lui-même les mots qu'il employait. Pour ce faire, nous avons utilisé un corpus de 119 textes d'élèves provenant d'une commission scolaire de la Rive-Sud de Montréal dont les enseignants participaient à une recherche-action. ${ }^{4}$ Une formation sur la grammaire actuelle et son enseignement a été offerte aux enseignants participants. Dans le but d'analyser les effets de cette formation sur les textes des élèves provenant de classes participantes (groupe expérimental) et non participantes (groupe témoin), nous avons demandé à un petit échantillon d'élèves d'écrire un texte d'environ une page sur un sujet de leur choix. Lors de l'analyse de l'étude principale (David et Giguère, 2016), notre attention a été retenue par les erreurs liées aux homophones grammaticaux et c'est la raison pour laquelle la présente recherche a émergé. Il s'agit donc d'une utilisation de données secondaires de recherche. Seuls les textes de début d'année scolaire, c'est-à-dire les textes écrits à l'automne 2010, 2011 et 2012 ont été utilisés pour cette étude. Il n'y a donc pas d'effet lié à la formation reçue par les enseignants puisqu'il s'agit des productions « prétest ».

\section{Sélection des participants}

Le corpus étudié a été constitué dans 36 classes de la fin du primaire et du début du secondaire. Afin de réaliser à la fois la production de textes et des entretiens métagraphiques auprès d'élèves, ces derniers demandant du temps lors de la collecte et lors de l'analyse, le protocole visait à sélectionner de trois à cinq élèves par classe. Les enseignants ont choisi les élèves en sélectionnant au moins un élève faible, un élève moyen et un élève fort par groupe, selon la consigne donnée, pourvu que l'enfant soit en mesure de bien s'exprimer en entretien. Il est à noter que l'analyse des entretiens constitue l'objet d'un autre article (Giguère et Aldama, 2019). Ainsi, 119 élèves provenant de 36 classes dans 16 écoles différentes ont été choisis par les enseignants. Les parents de tous ces élèves avaient signé un formulaire de consentement de participation à une recherche incluant des utilisations futures de données. L'équilibre entre les sexes n'ayant pas été demandé dans la consigne, le nombre de garçons et de filles n'est pas équivalent. Le Tableau 1 montre la répartition des élèves selon le sexe, l'âge et le milieu socioéconomique de leur école. Pour les analyses selon les âges des élèves, nous avons resserré l'échantillon aux élèves de 10 et 11 ans provenant de classes de $5^{\mathrm{e}}$ et de $6^{\mathrm{e}}$ année du primaire puisqu'ils étaient en nombre plus important (en gras dans le tableau). Les autres élèves ne font donc pas partie de ce calcul $(N=102)$. 
Tableau 1

Répartition des élèves selon le sexe, l'âge et le milieu socioéconomique

\begin{tabular}{|c|c|c|c|c|c|c|c|}
\hline \multirow[b]{3}{*}{ Âge (ans) } & \multirow[b]{3}{*}{$N$} & & & \multicolumn{4}{|c|}{$\begin{array}{l}\text { Indice de milieu } \\
\text { socioéconomique }^{\mathrm{a}}\end{array}$} \\
\hline & & Sexe & & \multicolumn{2}{|c|}{$\begin{array}{l}N \text { élèves } \\
\text { provenant de } \\
\text { milieux } \\
\text { favorisés } \\
\text { (IMSE 1-2-4) }\end{array}$} & \multicolumn{2}{|c|}{$\begin{array}{l}N \text { élèves } \\
\text { provenant de } \\
\text { milieux } \\
\text { défavorisés } \\
\text { (IMSE 8-9) }\end{array}$} \\
\hline & & garçons & filles & Garçons & Filles & Garçons & Filles \\
\hline 9 & 3 & 2 & 1 & 0 & 0 & 2 & 1 \\
\hline 10 & 54 & 25 & 29 & 13 & 18 & 12 & 11 \\
\hline 11 & 48 & 18 & 30 & 13 & 23 & 5 & 7 \\
\hline 10-11 (classe combinée) & 11 & 5 & 6 & 4 & 4 & 1 & 2 \\
\hline 12 & 3 & 2 & 1 & 0 & 0 & 2 & 1 \\
\hline Sous-total $N$ & & 52 & 67 & 30 & 45 & 22 & 22 \\
\hline Total $N$ & 119 & & & & & & \\
\hline
\end{tabular}

Note. Nous avons resserré l'échantillon aux élèves de 10 et 11 ans provenant de classes de $5^{\mathrm{e}}$ et de $6^{\mathrm{e}}$ année du primaire puisqu'ils étaient en nombre plus important (en gras dans le tableau). Les autres élèves ne font donc pas partie de ce calcul $(N=102)$.

${ }^{a}$ Cet indice est constitué aux $2 / 3$ par la sous-scolarité de la mère et au $1 / 3$ par l'inactivité des parents l'année précédant le recensement

(http://www.education.gouv.qc.ca/enseignants/aide-et-soutien/milieux-defavorises/agirautrement/indice-de-milieu-socio-economique-imse/). Rappelons que la passation des tests s'est réalisée à l'automne.

\section{Instrument et procédure de collecte}

La collecte des données commençait par une présentation des consignes de la tâche d'écriture par la chercheuse ou l'auxiliaire de recherche dans un local à part. Il était alors expliqué aux élèves que leur participation à l'étude visait à étudier les stratégies qu'ils employaient durant la tâche d'écriture. On leur demandait ensuite d'écrire un texte sur un thème de leur choix afin de réduire la charge cognitive associée à l'écriture et ainsi permettre plus aisément d'ajouter la charge cognitive liée au protocole de pensée à voix haute. Ils devaient partager oralement (au crayon « magique » qui les enregistrait individuellement) toutes les stratégies qu'ils employaient pendant et après la rédaction. La chercheuse ou l'auxiliaire les assistait (en petits groupes) pour leur rappeler par moments de verbaliser leurs réflexions lorsqu'ils écrivaient. Un entretien métagraphique avait ensuite lieu au cours duquel la chercheuse demandait à l'élève d'expliquer comment il faisait pour s'assurer qu'il n'y avait pas d'erreur dans son texte (visant ainsi ses stratégies d'autocorrection). Aucune stratégie ou aucun thème particulier n'était spécifiquement visé. La séance de rédaction durait en moyenne 30 à 40 minutes, ce qui permettait de les réaliser à l'intérieur d'une période de l'horaire de classe. 


\section{Protocole d'analyse}

Un codage des textes a été mené manuellement sur chaque copie photocopiée afin d'identifier tous les homophones utilisés par les élèves de même que leur exactitude dans le contexte, soit sa graphie correcte ou erronée. Ceci a permis de calculer par la suite la fréquence des divers homophones dans chaque texte de même que leur taux de réussite.

Une grille a été élaborée à partir de listes provenant de matériel didactique récent, d'études mentionnées précédemment (Boivin et Pinsonneault, 2012 ; Champoux, 2015 ; McNicoll et Roy, 1985) et des homophones grammaticaux relevés dans le corpus. Nous avons fait le choix de considérer les homophones grammaticaux selon le point de vue des élèves, en nous basant sur les savoirs dont ils disposaient pour orthographier les mots qu'ils choisissaient, c'est-à-dire les séries d'homophones enseignées. Cette grille a été bonifiée au fur et à mesure du codage pour obtenir 22 séries d'homophones et leurs diverses possibilités. Un recodage a été effectué à chaque ajout d'une nouvelle série. Un dénombrement du nombre total de mots a aussi été réalisé par les assistantes de recherche afin d'établir les taux d'occurrence. Le Tableau 2 montre les 22 séries d'homophones utilisées par les élèves dans le corpus de 119 textes, les lignes représentant les séries et les colonnes, les différentes graphies possibles. L'ordre des séries est arbitraire.

Il est important de noter que les formes fléchies (p. ex. sais/sait) ont été fusionnées pour centrer notre attention sur le choix de l'homophone (c'est/s'est/ces/ses/sais ou sait) et non sur l'accord du verbe (sais ou sait) ou son temps verbal (c'est ou c'était). Dans ce dernier cas (c'était ou $s$ 'était), l'erreur homophonique se situe dans le choix du $c$ ou du $s$ et non dans le temps du verbe être, d'où son amalgame à la série [sc]. De plus, les formes identiques à l'oral et à l'écrit ont parfois été discriminées pour permettre une analyse plus fine des erreurs : par exemple, le déterminant et le pronom la ou les sont identiques, mais selon la classe, il pourrait y avoir plus ou moins d'erreurs sur une forme, d'où leur classement spécifique. Au moment de coder les textes, le code associé au mot écrit était saisi (chiffre et lettre), de même que sa réussite (1) ou son échec (2) dans ce contexte (p. ex. *j'ai parlé a Martin-> 1a-1 / 4a-2). 
Tableau 2

Homophones utilisés par les élèves dans leur texte

\begin{tabular}{|c|c|c|c|c|c|c|c|c|}
\hline $\begin{array}{l}\text { Numéro } \\
\text { d'homo- } \\
\text { phone }\end{array}$ & $\begin{array}{l}\text { Transcription } \\
\text { phonétique }\end{array}$ & $\mathrm{a}$ & $\mathrm{b}$ & $\mathrm{c}$ & $\mathrm{d}$ & $\mathrm{e}$ & $\mathrm{f}$ & $\mathrm{g}$ \\
\hline 1 & $\mathrm{e}$ & é & er & ez & é adj. & & & \\
\hline 2 & s̃̃ & son & sont & & & & & \\
\hline 3 & $\tilde{\jmath}$ & on & ont & & & & & \\
\hline 4 & $\mathrm{a}, \mathrm{a}$ & $\mathrm{a} / \mathrm{as}$ & à & & & & & \\
\hline 5 & $\mathrm{~s} \varepsilon$ & ces & ses & c'est & s'est & $\begin{array}{l}\text { sais/ } \\
\text { sait }\end{array}$ & c'était & s'était \\
\hline 6 & ma, ma & $\mathrm{ma}$ & m'a/m'as & & & & & \\
\hline 7 & la, la & là & l'a/l'as & la-dét & la-pron & & & \\
\hline 8 & $1 \varepsilon$, le & les -dét & l'est/l'es & l'ai & les-pron & & & \\
\hline 9 & ta, ta & ta & t'a/t'as & & & & & \\
\hline 10 & $\mathrm{sa}, \mathrm{sa}$ & ça & $\mathrm{Sa}$ & & & & & \\
\hline 11 & sə & ce-dét. & se & & & & & \\
\hline 12 & $\mathrm{u}$ & ou & où & & & & & \\
\hline 13 & lœr & leur & leurs & & & & & \\
\hline 14 & mõ & mon & m'ont & & & & & \\
\hline 15 & $\mathrm{~m} \varepsilon$ & mais & mes & m'est /m'es & $\begin{array}{l}\text { mets/ } \\
\text { met }\end{array}$ & & & \\
\hline 16 & $\mathrm{t} \varepsilon$ & tes & t'est/t'es & & & & & \\
\hline 17 & set & cet & cette & & & & & \\
\hline 18 & $\varepsilon$ & est/es & Ait & & & & & \\
\hline 19 & $\mathrm{p} \varnothing$ & $\begin{array}{l}\text { peux/ } \\
\text { peut }\end{array}$ & peu & & & & & \\
\hline 20 & ni & ni & n’y & & & & & \\
\hline 21 & si & si & s'y & & & & & \\
\hline 22 & syr & sur & sûr & & & & & \\
\hline
\end{tabular}




\section{Résultats}

Cette section présente d'abord les résultats d'occurrences des séries d'homophones identifiées dans les textes, suivis par les taux de réussite des élèves pour chacun d'eux.

\section{Occurrences des séries d'homophones}

Afin de répondre à la première question de recherche, les occurrences des différentes séries d'homophones ont été analysées. D'abord, sur les 12483 mots utilisés par les élèves dans leurs textes, 2441 mots sont considérés comme des homophones selon notre grille, soit $19,55 \%$ des mots. Les homophones grammaticaux étaient donc effectivement très fréquents dans les écrits des élèves, plus encore que ce qu'a rapporté Catach (1995, 6\%). Les mots homophones ont été relevés et dénombrés sans égard à la deuxième question de recherche à savoir si leur orthographe était correcte ou non. La série d'homophones ayant le plus d'occurrences dans les textes du corpus était celle des mots qui se terminaient en $-E$ (voir Tableau 3 ) pour une fréquence de $24,5 \%$, suivie par les a/à avec une proportion de 13,9\%. La plupart des séries subséquentes comportaient un déterminant dans la série analysée : là/l'a-l'as/la déterminant/la pronom avec une proportion de $9 \%$, les déterminant/l'est-l'es/l'ai/les pronom avec une proportion de 7,5\%, la série mais/mes/m'est-m'es/mets-met avec une proportion de $6,3 \%$, le couple $m a / m$ 'as-m'a avec une proportion de $6,2 \%$ et enfin le déterminant mon (aucune occurrence pour $m$ 'ont) avec une proportion de 5,4\%. Dans les couples montrant également une haute fréquence, on retrouvait la paire on/ont qui était présente dans une proportion de $5,7 \%$, est/ait dans une proportion de $4,5 \%$ et le couple ce/se dans une proportion de $4 \%$. Les 11 autres séries présentaient un poids négligeable dans le corpus (1,8\% et moins). Ainsi, les mots de forme homophone étaient fréquents dans les textes (près de $20 \%$ des mots), mais uniquement une dizaine de séries présentait une occurrence de plus de $2 \%$ du corpus.

Le Tableau 3 permet également d'apprécier la fréquence de chaque mot homophone dans sa série. Rappelons que ces résultats font voir les occurrences réalisées par les élèves, correctes ou non. De manière générale, les déterminants étaient beaucoup plus fréquents que les autres catégories grammaticales (en gras dans le tableau). Ceci est d'autant plus vrai lorsque l'on porte le regard sur les formes disjointes, c'est-à-dire les verbes précédés d'un pronom complément ( $m$ 'ont, $t$ 'est, $m$ 'est, etc.) dont l'occurrence était assez rare dans les textes de notre corpus. En effet, le déterminant la présentait 195 occurrences sur un total de 219 formes homophones de [la, la ] pour une proportion de $89 \%$, le déterminant les, 168 occurrences sur un total de 182 formes homophones de [le] pour une proportion de $92 \%$ et le déterminant ma est revenu à 142 reprises sur un total de 151 formes homophones de [ma, ma ], soit $94 \%$ des occurrences. Le déterminant mes a montré 89 occurrences sur 153 formes homophones de [me] pour une proportion de $58 \%$ alors que la conjonction, mais occupait quand même 39,2 \% des occurrences de la série. Enfin, le déterminant mon est revenu 133 fois sur 133, donc $100 \%$ des occurrences tout comme le déterminant ta dont les 5 occurrences représentaient également $100 \%$ de cette série. Concernant les autres séries, est a montré une fréquence largement plus grande que ait $(98 \%)$, le pronom on était plus fréquent que le verbe ou l'auxiliaire avoir ont $(77,5 \%)$ et la paire ce/se se partageait les occurrences avec une certaine prépondérance pour le pronom se $(58 \%)$. Ainsi, les déterminants étaient les mots homophones les plus fréquents dans les différentes séries analysées. 
Tableau 3

Séries d'homophones présentées selon leur ordre de fréquence décroissant

\begin{tabular}{|c|c|c|c|c|c|c|c|c|c|c|}
\hline $\begin{array}{l}\text { Numéro } \\
\text { d'homophone } \\
\text { selon la grille }\end{array}$ & $\begin{array}{l}\text { Transcription } \\
\text { phonétique }\end{array}$ & Séries d'l & Iones et occ & nces dans le o & & & & & Total & $\%$ \\
\hline 1 & $\mathrm{e}$ & $\begin{array}{l}192 \\
\text { é }\end{array}$ & $\begin{array}{l}334 \\
\text { er }\end{array}$ & $\begin{array}{l}15 \\
\mathrm{ez}\end{array}$ & $\begin{array}{l}56 \\
\text { é adj }\end{array}$ & & & & 597 & 24,5 \\
\hline 4 & $\mathrm{a}, \mathrm{a}$ & $\begin{array}{l}146 \\
\text { a/as }\end{array}$ & $\begin{array}{l}193 \\
\text { à }\end{array}$ & & & & & & 339 & 13,9 \\
\hline 7 & la, la & $\begin{array}{l}14 \\
\text { là }\end{array}$ & $\begin{array}{l}3 \\
\text { l'a/l'as }\end{array}$ & $\begin{array}{l}195 \\
\text { la (dét) }\end{array}$ & $\begin{array}{l}7 \\
\text { la (pron) }\end{array}$ & & & & 219 & 9 \\
\hline 8 & $1 \varepsilon$ & $\begin{array}{l}168 \\
\text { les (dét) }\end{array}$ & $\begin{array}{l}0 \\
\text { l'est/l'es }\end{array}$ & $\begin{array}{l}3 \\
\text { l'ai }\end{array}$ & $\begin{array}{l}11 \\
\text { les (pron) }\end{array}$ & & & & 182 & 7,5 \\
\hline 15 & $\mathrm{~m} \varepsilon$ & $\begin{array}{l}60 \\
\text { mais }\end{array}$ & $\begin{array}{l}89 \\
\text { mes }\end{array}$ & $\begin{array}{l}1 \\
\text { m'est/m'es }\end{array}$ & $\begin{array}{l}3 \\
\text { met/mets }\end{array}$ & & & & 153 & 6,3 \\
\hline 6 & $\mathrm{ma}, \mathrm{ma}$ & $\begin{array}{l}142 \\
\mathrm{ma}\end{array}$ & $\begin{array}{l}9 \\
\text { m'a/m'as }\end{array}$ & & & & & & 151 & 6,2 \\
\hline 3 & $\tilde{\jmath}$ & $\begin{array}{l}107 \\
\text { on }\end{array}$ & $\begin{array}{l}31 \\
\text { ont }\end{array}$ & & & & & & 138 & 5,7 \\
\hline 14 & mõ & $\begin{array}{l}133 \\
\text { mon }\end{array}$ & $\begin{array}{l}0 \\
\text { m'ont }\end{array}$ & & & & & & 133 & 5,4 \\
\hline 18 & $\varepsilon$ & $\begin{array}{l}109 \\
\text { est }\end{array}$ & $\begin{array}{l}2 \\
\text { ait }\end{array}$ & & & & & & 111 & 4,5 \\
\hline 11 & sə & $\begin{array}{l}41 \\
\text { ce (dét) }\end{array}$ & $\begin{array}{l}57 \\
\text { se }\end{array}$ & & & & & & 98 & 4 \\
\hline 5 & $\mathrm{~s} \varepsilon$ & $\begin{array}{l}7 \\
\text { ces }\end{array}$ & $\begin{array}{l}12 \\
\text { ses }\end{array}$ & $\begin{array}{l}47 \\
\text { c'est }\end{array}$ & $\begin{array}{l}4 \\
\text { s'est }\end{array}$ & $\begin{array}{l}4 \\
\text { sais/sait }\end{array}$ & $\begin{array}{l}14 \\
\text { c'était }\end{array}$ & $\begin{array}{l}6 \\
\text { s'était }\end{array}$ & 94 & 3,9 \\
\hline
\end{tabular}




\begin{tabular}{|c|c|c|c|c|c|}
\hline $\begin{array}{l}\text { Numéro } \\
\text { d'homophone } \\
\text { selon la grille }\end{array}$ & $\begin{array}{l}\text { Transcription } \\
\text { phonétique }\end{array}$ & \multicolumn{2}{|c|}{ Séries d'homophones et occurrences dans le corpus } & Total & $\%$ \\
\hline 17 & set & $\begin{array}{l}6 \\
\text { cet }\end{array}$ & $\begin{array}{l}38 \\
\text { cette }\end{array}$ & 44 & 1,8 \\
\hline 2 & s̃̃ & $\begin{array}{l}15 \\
\text { son }\end{array}$ & $\begin{array}{l}25 \\
\text { sont }\end{array}$ & 40 & 1,6 \\
\hline 10 & sa, sa & $\begin{array}{l}11 \\
\text { ça }\end{array}$ & $\begin{array}{l}19 \\
\text { sa }\end{array}$ & 30 & 1,2 \\
\hline 12 & $\mathrm{u}$ & $\begin{array}{l}25 \\
\text { ou }\end{array}$ & $\begin{array}{l}5 \\
\text { où }\end{array}$ & 30 & 1,2 \\
\hline 22 & syr & $\begin{array}{l}21 \\
\text { sur }\end{array}$ & $\begin{array}{l}1 \\
\text { sûr }\end{array}$ & 22 & 0,9 \\
\hline 19 & $\mathrm{p} \varnothing$ & $\begin{array}{l}14 \\
\text { peux/peut }\end{array}$ & $\begin{array}{l}8 \\
\text { peu }\end{array}$ & 22 & 0,9 \\
\hline 13 & lœr & $\begin{array}{l}5 \\
\text { leur }\end{array}$ & $\begin{array}{l}7 \\
\text { leurs }\end{array}$ & 12 & 0,5 \\
\hline 21 & si & $\begin{array}{l}11 \\
\text { si }\end{array}$ & $\begin{array}{l}0 \\
\text { s'y }\end{array}$ & 11 & 0,5 \\
\hline 16 & t $\varepsilon$ & $\begin{array}{l}4 \\
\text { tes }\end{array}$ & $\begin{array}{l}2 \\
\text { t'est/t'es }\end{array}$ & 6 & 0,2 \\
\hline 20 & ni & $\begin{array}{l}1 \\
\text { ni }\end{array}$ & $\begin{array}{l}3 \\
\text { n'y }\end{array}$ & 4 & 0,2 \\
\hline 9 & ta, ta & $\begin{array}{l}5 \\
\text { ta }\end{array}$ & $\begin{array}{l}0 \\
t^{\prime} a / t^{\prime} \text { as }\end{array}$ & 5 & 0,2 \\
\hline Total & & & & 2441 & 100 \\
\hline
\end{tabular}

Note. Les déterminants (en gras dans le tableau) étaient beaucoup plus fréquents que les autres catégories grammaticales. 
La prochaine section présentera les taux de réussite pour chacune des séries de mots homophones, de même que pour chaque homophone dans sa série.

\section{Taux de réussite des différents types d'homophones}

Afin de répondre à la deuxième question de recherche, une analyse des réussites et des erreurs des élèves a été menée. Cette sous-section s'attarde à la présentation des taux de réussites des élèves selon les séries d'homophones, mais également selon leurs différentes graphies possibles. Le Tableau 4 illustre ces taux de réussite.

Tableau 4

Occurrences, nombre et pourcentage de réussite des séries de mots homophones identifiés dans le corpus de 119 textes d'élèves selon un ordre décroissant

\begin{tabular}{|c|c|c|c|c|}
\hline $\begin{array}{l}\text { Numéro } \\
\text { d'homophone } \\
\text { selon la grille }\end{array}$ & $\begin{array}{l}\text { Transcription } \\
\text { phonétique }\end{array}$ & Occurrences & $\mathrm{Nb}$ réussis & $\%$ de réussite \\
\hline 8 & $1 \varepsilon$ & 182 & 182 & 100 \\
\hline 14 & mว̃ & 133 & 133 & 100 \\
\hline 20 & ni & 4 & 4 & 100 \\
\hline 21 & si & 11 & 11 & 100 \\
\hline 22 & syr & 22 & 22 & 100 \\
\hline 7 & la, la & 219 & 218 & 99,5 \\
\hline 17 & set & 44 & 43 & 97,7 \\
\hline 6 & $\mathrm{ma}, \mathrm{ma}$ & 151 & 147 & 97,4 \\
\hline 18 & $\varepsilon$ & 111 & 107 & 96,4 \\
\hline 15 & $\mathrm{~m} \varepsilon$ & 153 & 147 & 96,1 \\
\hline 19 & pø & 22 & 21 & 95,5 \\
\hline 2 & s̃̃ & 40 & 38 & 95 \\
\hline 3 & $\tilde{\jmath}$ & 138 & 122 & 88,4 \\
\hline 4 & $a, a$ & 339 & 294 & 86,7 \\
\hline 11 & sə & 98 & 85 & 86,7 \\
\hline 12 & $\mathrm{u}$ & 30 & 26 & 86,7 \\
\hline 1 & e & 597 & 511 & 85,6 \\
\hline 13 & lœr & 12 & 10 & 83,3 \\
\hline 9 & ta, ta & 5 & 4 & 80 \\
\hline 5 & $\mathrm{~S} \varepsilon$ & 94 & 74 & 78,7 \\
\hline 10 & $\mathrm{sa}, \mathrm{sa}$ & 30 & 22 & 73,3 \\
\hline 16 & $\mathrm{t} \varepsilon$ & 6 & 4 & 66,7 \\
\hline Total & & 2441 & 2225 & 91,2 \\
\hline
\end{tabular}

Ainsi, dans l'ensemble des 22 séries d'homophones identifiées dans notre corpus, cinq séries ont montré $100 \%$ de réussite par les élèves, c'est-à-dire que toutes les occurrences 
étaient exactes. Il s'agissait des [le] (les déterminant, l'est-l'es, l'ai, les pronom), du déterminant mon (aucune occurrence pour m'ont), des [ni] (ni-n'y), [si] (si-s'y) et [syr] (sur, sûr).

En revanche, trois séries ont attiré notre attention pour leur faible taux de réussite : [tz] (tes,t'est-t'es) avec 66,7\%, [sa, sa] (sa,ça) avec 73,3\% et la série ces, ses, c'est, s'est, sait-sais, c'était, s'était avec un taux de réussite de 78,7\%. Les deux premières séries ont montré une fréquence négligeable dans notre corpus, soit respectivement $0,2 \%$ et $1,2 \%$, ce qui en a diminué la valeur statistique. Néanmoins, en ce qui concerne la paire [t $\varepsilon]$, le déterminant tes a été réussi 4 fois sur 4, mais la forme disjointe, 0 sur 2 . Ainsi, à deux reprises, un élève a utilisé une forme disjointe en position de déterminant comme dans l'exemple suivant : "* T'es blagues me font beaucoup rire » (élève 77). Quant à la série [sa, sa], les élèves ont choisi le présentatif ça correctement 10 fois sur 11, alors qu'ils ont opté pour le déterminant $s a$ en place du présentatif ça 7 fois sur 19, comme dans le contexte «* Sa m’a fait très mal » (élève 5).

Considérant que seule la série $[\mathrm{s} \varepsilon$ ] étant à la fois un peu plus fréquente $(3,9 \% \mathrm{du}$ corpus) et moins bien réussie (78,7\% de réussite), nous avons examiné les occurrences posant problème aux élèves. Comme le montre le Tableau 5, c'étaient les graphies pronominales qui occasionnaient le plus de problèmes aux élèves ( $s$ 'est et $s$ 'était), de même que le déterminant possessif (ses) ; les présentatifs (c'est et c'était) étaient les formes les plus souvent rencontrées et étaient presque toujours réussis. C'est donc dire que les élèves utilisaient souvent les formes s'est et ses incorrectement, comme dans «*Le pire, s'est quand [la neige] fond et que c'est mouillé partout » (élève 18) ou encore dans «* . . alors ses a se moment quil avais trouver une trappe dans le plancher » (élève 90).

Tableau 5

Fréquence, occurrence, nombre et pourcentage de réussite des homophones liés à la série [sc] dans le corpus de 119 textes d'élèves

\begin{tabular}{lllll}
\hline $\begin{array}{l}\text { Graphies possibles } \\
\text { dans la série } \\
\text { (ordre aléatoire) }\end{array}$ & $\begin{array}{l}\text { Fréquence } \\
\text { dans la série } \\
\text { (en \%) }\end{array}$ & Occurrences & Nb réussis & \% de réussite \\
\hline Ces & 7,4 & 7 & 5 & 71,4 \\
Ses & $\mathbf{1 2 , 7}$ & $\mathbf{1 2}$ & $\mathbf{6}$ & $\mathbf{5 0}$ \\
C'est & 50 & 47 & 43 & 91,5 \\
S'est & $\mathbf{4 , 3}$ & $\mathbf{4}$ & $\mathbf{2}$ & $\mathbf{5 0}$ \\
Sais-sait & 4,3 & 4 & 3 & 75 \\
C'était & 14,9 & 14 & 14 & 100 \\
S'était & $\mathbf{6 , 4}$ & $\mathbf{6}$ & $\mathbf{1}$ & $\mathbf{1 6 , 7}$ \\
\hline
\end{tabular}

Note. Les items en gras représentent les \% de réussite les moins élevés.

Ainsi, le portrait des formes les moins réussies se précise : la forme disjointe de $t^{\prime} a$ utilisée comme $t a$, $s a$ utilisé en place de ça et les formes $s$ 'est/s'était de même que ses étaient choisies au lieu de c'est ou c'était.

Malgré la réussite relative des autres séries d'homophones, nous avons détaillé, parmi les séries les plus fréquentes, lesquelles posaient le plus problème aux élèves. Ainsi, par ordre décroissant de fréquence, les finales en [e] sont réussies à 85,6\% et le couple [a, a] est réussi à $87,6 \%$. Nous n'avons cependant pas inclus dans le tableau les séries [la] et [lع] puisqu'elles présentaient des taux de réussite de $99,5 \%$ et de $100 \%$. Venaient 
ensuite les [̃̃ ] et [sə]. Ainsi, nous pouvons constater à la lecture du Tableau 6 que ce sont les formes verbales qui représentent les items les moins réussis par les élèves dans les séries ([e], [a, a], [̃] ) de même que le pronom se (dont la position syntaxique se situe dans le groupe verbal $[\mathrm{GV}]$ selon sa fonction-complément direct ou indirect du verbe).

Tableau 6

Fréquence, occurrence, nombre et pourcentage de réussite des homophones liés aux séries les plus fréquentes dans le corpus de 119 textes d'élèves

\begin{tabular}{llllll}
\hline $\begin{array}{l}\text { Graphies } \\
\text { possibles dans } \\
\text { les séries [e], } \\
\text { [a, a ], [̃ ] et }\end{array}$ & $\begin{array}{l}\text { Fréquence } \\
\text { dans la série } \\
\text { (en \%) }\end{array}$ & $\begin{array}{l}\text { Taux de } \\
\text { réussite } \\
\text { (en \%) }\end{array}$ & Occurrences & Nb réussis & $\begin{array}{l}\% \text { de } \\
\text { réussite }\end{array}$ \\
\hline É & 32 & 85,6 & 192 & 174 & 90,6 \\
Er & 56 & & 334 & 274 & 82 \\
Ez & 2,5 & & 15 & 8 & 53,5 \\
É (adj) & 9,4 & & 56 & 55 & 98,2 \\
A-as & 43 & 87,6 & 146 & 109 & 74,7 \\
$\dot{A}$ & 57 & & 193 & 185 & 95,9 \\
On & 77,5 & 88,4 & 107 & 104 & 97,2 \\
Ont & 22,5 & & 31 & 18 & 58,1 \\
Ce & 42 & 86,7 & 41 & 39 & 95,1 \\
Se & 58 & & 57 & 46 & 80,7 \\
\hline
\end{tabular}

À la suite de l'analyse de cette série, nous avons tenté de savoir si certains contextes présentant des erreurs pour les [a, a] et les finales en [e] étaient reliés entre eux, par exemple dans les temps composés avec l'auxiliaire avoir. Cette hypothèse s'est avérée juste, car sur un ensemble de 117 contextes d'erreurs de ces deux types, 33 se trouvaient dans un contexte syntaxique auxiliaire avoir + participe passé (soit $28 \%$ des erreurs [a, a], [e] combinées). Sur ce nombre, 20 erreurs dans ce contexte portaient sur le participe passé écrit comme un infinitif par l'élève (*J'ai diner [élève 107]). Il s'agissait de l'erreur la plus fréquente dans cette combinaison. L'inverse n'était pas vrai puisque seulement cinq erreurs concernent la préposition à suivie d'un infinitif en -er (soit $5 \%$ ) comme dans «*Il commence à volé chez ces voisins » (élève 41). En somme, il semble que les élèves préféraient l'infinitif et que la construction du passé composé leur posait problème puisqu'il s'agissait de la combinaison la plus manquée des formes [e], [a, a].

\section{Taux de réussite des formes homophones selon les caractéristiques des élèves}

Dans le but de répondre à la troisième question de recherche, une analyse des caractéristiques des élèves et de leurs réussites a été menée. Si l'on s'attarde au taux de réussite selon le sexe des élèves (Tableau 7), ce sont les filles qui performaient significativement mieux que les garçons $(p=, 002)$ de même que les élèves de milieux favorisés comparativement aux élèves provenant de milieux défavorisés $(p=, 002)$. Les élèves de 11 ans semblaient mieux performer que les élèves de 10 ans, mais cette différence n'est pas significative $(p=, 350)$. Rappelons que nous avons éliminé de ce dernier calcul les 
élèves fréquentant une classe combinée (10-11 ans) et les élèves de 9 et de 12 ans pour ne conserver que 102 élèves.

Tableau 7

Pourcentage de réussite de l'orthographe des mots homophones des élèves selon leur milieu socioéconomique, leur sexe et leur âge

\begin{tabular}{lllllllll}
\hline $\begin{array}{l}\text { Sous- } \\
\text { groupes }\end{array}$ & $N$ & Min & $25 \%$ & Médiane & $75 \%$ & Max & $M$ & $E-T$ \\
\hline Défavorisé & 44 & 50 & 83 & 91 & 97 & 100 & 88 & 12 \\
Favorisé & 73 & 57 & 92 & 100 & 100 & 100 & 95 & 8 \\
Filles & 66 & 58 & 92 & 100 & 100 & 100 & 95 & 8 \\
Garçons & 51 & 50 & 86 & 92 & 96 & 100 & 89 & 12 \\
10 ans & 54 & 50 & 86 & 95 & 100 & 100 & 91 & 12 \\
11 ans & 48 & 63 & 91 & 95 & 100 & 100 & 93 & 9 \\
\hline
\end{tabular}

L'analyse présentée au Tableau 7 montre que la performance des garçons $(\mu=89)$ est équivalente à celle d'élèves provenant de milieux défavorisés $(\mu=88)$ et que celle des filles $(\mu=95)$ est plutôt équivalente à celle d'élèves de milieux favorisés $(\mu=95)$. Pour ce qui est de l'âge, les élèves des deux sexes sont plus faibles à 10 ans $(\mu=91)$ qu'à 11 ans $(\mu=93),{ }^{5}$ mais au moins la moitié des élèves réussit quand même bien (médiane équivalente de 95 entre 10 et 11 ans). On remarque aussi que l'écart-type est toujours plus grand chez les sous-groupes plus faibles (milieux défavorisés, garçons, 10 ans).

Pour mieux comprendre l'influence de ces trois facteurs, c'est-à-dire le milieu, le sexe et l'âge des élèves, le Tableau 8 présente les pourcentages de réussite selon les caractéristiques combinées entre les populations. À la lecture de ce tableau, il est à relever que 12 élèves présentent un triple désavantage : il s'agit des garçons de 10 ans issus de milieux défavorisés et effectivement, ces élèves obtiennent une moyenne de $84 \%$ avec un écart-type de 15. Cependant, les moyennes montrent une plus grande difficulté chez les garçons de 11 ans en milieu défavorisé (bien qu'ils ne soient que cinq) : aucun ne parvient à orthographier correctement tous les homophones de son texte, alors que la moitié des filles de milieu favorisé (de 11 ans aussi bien que de 10 ans) le font.

Tableau 8

Pourcentage de réussite de l'orthographe des mots homophones selon la combinaison de leur âge, leur sexe et leur milieu socioéconomique

\begin{tabular}{lllllllllll}
\hline Âge & Sexe & IMSE & $N$ & Min & $25 \%$ & Médiane & $75 \%$ & Max & $M$ & $E-T$ \\
\hline 10 ans & Filles & Déf & 11 & 79 & 89 & 96 & 100 & 100 & 93 & 8 \\
& & Fav & 18 & 58 & 94 & 100 & 100 & 100 & 95 & 11 \\
& \multirow{4}{*}{ Garçons } & Déf & 12 & 50 & 76 & 89 & 94 & 100 & 84 & 15 \\
& & Fav & 13 & 57 & 86 & 92 & 100 & 100 & 89 & 12 \\
& \multirow{4}{*}{ Filles } & Déf & 7 & 68 & 79 & 91 & 95 & 100 & 87 & 12 \\
& & Fav & 23 & 90 & 98 & 100 & 100 & 100 & 98 & 3 \\
& \multirow{2}{*}{ Garçons } & Déf & 5 & 63 & 68 & 81 & 94 & 97 & 81 & 15 \\
& & Fav & 13 & 87 & 90 & 94 & 95 & 100 & 93 & 5 \\
\hline
\end{tabular}

Note. IMSE $=$ Indice de milieu socioéconomique. 
En conclusion, l'analyse de notre corpus montre que les caractéristiques de sexe (les garçons), d'âge (10 ans) et de milieu socioéconomique (milieux défavorisés) sont des conditions qui influencent les habiletés des élèves en orthographe des homophones grammaticaux.

\section{Discussion}

Afin de répondre à la première question de recherche, les séries homophoniques les plus fréquentes ont été identifiées par un rapport d'occurrence des mots de formes homophones sur l'ensemble des mots du corpus de 119 textes, de même que les fréquences des homophones les composant. Ainsi, les résultats montrent d'abord que les mots homophones composent environ $20 \%$ de notre corpus et sont donc très utilisés par les élèves de 9 à 12 ans, mais uniquement pour une dizaine de séries. Plus spécifiquement, les finales en $/ E$ / et le couple $a / a ̀$ sont les plus fréquents. Ils constituent environ le tiers des homophones identifiés dans le corpus. Ces résultats vont dans le même sens que ceux de Champoux (2015) pour les finales en /E/ et que McNicoll et Roy (1985) pour le couple $a / \grave{a}$. De plus, comme l'ont identifié McNicoll et Roy (1985), les formes simples sont plus fréquentes que les formes disjointes : 850 formes simples ont été comptabilisées pour 123 formes disjointes ou présentant un signe diacritique. Les déterminants sont également largement plus fréquents que les autres catégories grammaticales dans les différentes séries.

Les didacticiens du français, particulièrement en ce qui concerne la grammaire, préconisent depuis plusieurs années l'enseignement des régularités de la langue et la compréhension du système plutôt que la maîtrise de ses exceptions (Brissaud et Cogis, 2011 ; Nadeau et Fisher, 2006). Ainsi, il apparaît pertinent pour les enseignants de travailler d'abord les groupes du nom (où se trouvent les déterminants) avec les élèves plus jeunes pour consolider leur réussite de même que la conjugaison des verbes avec les élèves plus vieux, particulièrement en ce qui concerne le fonctionnement du système verbal. Il apparait important d'amener les élèves à discerner les différences entre les notions d'infinitif et de verbe conjugué, de temps simples et de temps composés, de même que d'auxiliaire et de verbe. Ce faisant, les élèves seraient davantage en mesure de discriminer en contexte les verbes conjugués, les infinitifs, les auxiliaires et les participes passés et ce, peu importe leur prononciation, afin de bien les orthographier. Comme nos résultats montrent des lacunes sur les formes verbales présentes dans les différentes séries, il serait opportun d'en faire un enseignement précis. Les travaux de Gauvin et Boivin (2013) de même que Gourdet, Cogis et Roubaud (2016) et Roy-Mercier et Chartrand (2016) peuvent orienter le travail des enseignants à ce sujet. Notons que ce concept lié aux temps composés est présenté au $3^{\mathrm{e}}$ cycle du primaire dans la Progression des apprentissages (MELS, 2009) : c'est peut-être la raison pour laquelle les élèves éprouvent des difficultés avec les finales en [e]. En effet, selon le curriculum, certains élèves de notre corpus (10 ans) avaient étudié l'identification de l'infinitif, mais n'avaient pas encore étudié formellement les temps composés. Ces résultats semblent montrer un retard face à ceux de Brissaud et coll. (2006), en raison de la fréquence plus grande des infinitifs dans notre corpus de textes d'élèves de 10 et 11 ans. Ce décalage pourrait orienter certaines pratiques d'enseignement des temps composés au $3^{\mathrm{e}}$ cycle du primaire.

La deuxième question de recherche s'intéressait aux réussites des élèves lorsqu'il s'agissait d'orthographier des mots homophones qu'ils choisissaient d'utiliser dans des textes libres. Les analyses indiquent que la très grande majorité des homophones de notre 
corpus a été bien écrite par les élèves, soit 91,2\%. Notre échantillon ne nous permet cependant pas de généraliser ces résultats ou de connaître le poids des erreurs d'homophones par rapport à l'ensemble des erreurs d'orthographe des élèves puisqu'aucune analyse statistique n'a été menée sur les autres formes d'erreurs dans cette étude. Cependant, à la lumière de nos analyses, la situation ne semble pas si désastreuse que nous l'avions anticipé : si on ne s'attarde qu'à ce pourcentage $(91,2 \%)$, il est difficile d'y voir un problème d'orthographe. La prise en compte des réussites de tous les mots des séries explique sans doute ce résultat : les analyses des mots erronés met bien souvent de côté les réussites, somme toute nombreuses selon notre corpus.

Certes, des erreurs plus fréquentes ont été répertoriées : la forme disjointe $t^{\prime} a$ utilisée comme $t a$, sa utilisé en place de ça et les formes $s$ 'est/s'était de même que ses sont choisies au lieu de c'est ou c'était. Il en est de même pour la combinaison $a$ auxiliaire et -é participe passé, donc l'orthographe du passé composé, qui est plus manquée que la combinaison préposition à et verbe à l'infinitif -er, mieux réussie. Certains penseront que les pratiques d'enseignement des trucs fonctionnent et qu'il n'est pas nécessaire de les modifier puisqu'elles semblent porter leurs fruits. D'autres, en revanche, se poseront la question : «Comment justifier tout ce temps d'enseignement consacré spécifiquement à cette problématique grammaticale de la langue qui n'est pas au programme de formation?»

Bien entendu, les autres résultats présentés dans cet article mènent à une tout autre conclusion et suggèrent un véritable changement dans les pratiques d'enseignement des homophones dans les classes puisque certaines populations s'avèrent plus vulnérables : les garçons de 10 ans provenant de milieux défavorisés. Un travail sur les classes de mots ( $\mathrm{p}$. ex. sur les déterminants possessifs comme mon, ton, son, ta, etc. en raison des formes concurrentes pronom-verbe : $m$ 'ont, $t$ 'ont, $t^{\prime}$ a ou verbe : sont ), sur la conjugaison et sur les temps verbaux influençant les finales en [e] (infinitif, temps composés, impératif, futur proche et les semi-auxiliaires) jumelé à une analyse des contextes de chaque phrase pour identifier chaque structure particulière nous apparait alors plus avisé (Boivin et Pinsonneault, 2012, 2018 ; Fisher, 1994 ; Nadeau et Fisher, 2006). Cette approche vise ainsi à amener les élèves à comprendre le système de la langue plutôt que d'utiliser les trucs et raccourcis qu'on leur propose et qu'ils utilisent parfois à tort. Il importe également d'éviter de traiter les mots comme étant des homophones, offrant ainsi aux élèves des exemples qui leur permettent de structurer leur savoir autour de ce concept « inventé » par l'école (Tallet, 2016b) et dont les choix didactiques, les listes enseignées, reposent sur des choix arbitraires des enseignants. Il serait préférable de traiter uniquement des exemples de phrases et les catégories grammaticales qui les construisent.

Contrairement à ce qu'on retrouve dans la littérature scientifique ailleurs dans la francophonie, les études québécoises publiées à propos de l'usage effectif des homophones grammaticaux ou sur la description des occurrences et des erreurs dans les textes sont peu nombreuses pour le primaire (Boivin et Pinsonneault, 2012, 2018 ; Champoux, 2015 ; McNicoll et Roy, 1985). Pourtant, les études traitant de l'enseignement problématique qu'on en fait à l'école (Brissaud et Cogis 2011 ; Cogis, 2005 ; Fisher, 1994 ; Nadeau et Fisher, 2006) ont confirmé que les élèves éprouvaient bel et bien des difficultés liées à ce phénomène orthographique. Notre corpus relativement restreint (119 élèves provenant d'une commission scolaire) et nos choix de séries d'homophones restreignent les possibilités d'analyse. En effet, nos résultats divergent probablement en raison du choix d'inclure les déterminants (largement réussis) dans les listes d'homophones. De plus, les 
textes des élèves ne permettaient pas de vérifier s'ils maitrisaient les formes concurrentes comme cela aurait été le cas avec des dictées ou des exercices imposés, par exemple. De plus, l'utilisation de données secondaires de recherche (Dionne et Fleuret, 2016) nous a contraintes à effectuer notre analyse sur des textes pour lesquels aucune consigne sur le genre du texte ou sur le type n'avait été donnée. Les textes produits ne sont donc pas nécessairement comparables entre eux dans le sens où le genre ou le type a pu influencer les choix linguistiques effectués et ainsi modifier le portrait des occurrences et des fréquences d'erreurs. Enfin, la persistance des interventions liées aux homophones grammaticaux chez les enseignants pourrait s'expliquer par la croyance des enseignants quant au taux d'erreurs de ce type que les élèves laissent dans leurs textes. Que ces erreurs, toutefois bien réelles, proviennent d'une difficulté inhérente à la langue écrite combinée ou pas à un enseignement didactiquement discutable (basé sur le rapprochement de paires et l'enseignement de trucs dans des textes troués), le portrait actuel que dresse cette étude semble pertinent pour éclairer les praticiens dans le milieu de l'enseignement au Québec.

\section{Conclusion}

Ainsi, 119 textes d'élèves de 9 à 12 ans fréquentant des écoles d'une commission scolaire de la Rive-Sud de Montréal ont été analysés au regard des homophones grammaticaux. Les résultats montrent que $19,55 \%$ des mots des textes étaient des homophones selon une grille de 22 séries. Ils sont donc effectivement fréquents dans des textes d'élèves. Parmi les plus fréquents, on compte les finales en [e], le couple [a, a] et les déterminants la et les. Sans égard à la fréquence, parmi les plus réussis, la série des [1ع], des [mõ] et les formes [ni], [si] et [syr]; parmi les moins réussis, la série de [t $\varepsilon$ ], le couple $[\mathrm{sa}, \mathrm{sa}]$ et la série des [sc]. Ces constantes vont dans le même sens que des études précédentes portant sur ces formes. Une analyse plus poussée a montré que les formes verbales et pronominales sont moins réussies par les élèves de la fin primaire que les autres formes d'homophones du corpus.

Enfin, malgré l'échantillon plutôt restreint ne permettant pas de généraliser nos résultats, nous pouvons avancer que le contexte socioéconomique de l'élève a une influence sur les résultats. Les garçons de milieux défavorisés réussissent moins bien à orthographier les homophones grammaticaux dans leurs textes. Une analyse des stratégies utilisée par les élèves devra être menée pour savoir à quels facteurs on peut attribuer ces difficultés (Giguère et Aldama, 2019).

Ces résultats sont de nature à orienter les pratiques scolaires en ciblant de réelles difficultés dans l'enseignement de l'orthographe tout en délaissant certaines formes dont ni l'occurrence ni les erreurs ne sont fréquentes. Les choix arbitraires des enseignants n'ont plus leur raison d'être. Il est temps de mettre fin à la pression sociale et scolaire de se conformer à l'enseignement de ce contenu associé à des séries à distinguer qui est devenu plus culturel que didactique.

\section{Notes}

${ }^{1}$ Bien qu'il arrive que certains homophones soient constitués de deux mots ( $m$ 'ont, $t$ 'a, etc.), nous conserverons l'expression « mot » pour parler des homophones afin de faciliter la lecture. 
${ }^{2}$ Recherche financée par le Programme d'aide à la recherche pour les nouveaux professeurs de la Faculté des sciences de l'éducation de l'Université du Québec à Montréal (UQAM).

${ }^{3}$ Selon les réalités sociolinguistiques, les mots peuvent se prononcer de manière identiques ou semblables. Par exemple, Brissaud et coll. (2006) ont analysé comme formes verbales homophones les é (participe passé), er (infinitif) et ais- $t$ (imparfait). Au Québec, l'amalgame de l'imparfait n'est pas considéré comme une forme homophone. De plus, comme l'ont indiqué McNicoll et Roy (1985), les Québécois prononcent différemment les $a / a /$ et $\grave{a} / a /$, ce qu'ils appellent les faux homophones.

${ }^{4}$ Dans le cadre d'un projet de recherche financé par le MELS-FRQSC, Programme de recherche sur l'écriture et la lecture. 2011-AC-137017.

${ }^{5}$ Rappelons que la passation des tests s'est réalisée à l'automne.

\section{Références}

Boivin, M.-C. et Pinsonneault, R. (2012). L'orthographe des homophones : une approche syntaxique. Lettre de l'AIRDF, 52, 36-40.

Boivin, M.-C. et Pinsonneault, R. (2014). Étude sur les erreurs de syntaxe, d'orthographe grammaticale et d'orthographe lexicale des élèves québécois en contexte de production écrite. Rapport de recherche déposé au ministère de l'éducation, du loisir et du sport du Québec, décembre 2014. Non disponible en ligne.

Boivin, M.-C. et Pinsonneault, R. (2018). Les erreurs de syntaxe, d'orthographe grammaticale et d'orthographe lexicale des élèves québécois en contexte de production écrite. Canadian Journal of Applied Linguistics, 21(1), 43-70. https://doi.org/10.7202/1050810ar

Brissaud, C., Chevrot, J.-P. et Lefrançois, P. (2006). Les formes verbales homophones en $/ \mathrm{E} /$ entre 8 et 15 ans : contraintes et conflits dans la construction des avoirs sur une difficulté orthographique. Langue française, 151, 74-93.

Brissaud, C. et Cogis, D. (2011). Comment enseigner l'orthographe aujourd'hui. Paris, France : Hatier.

Catach, N. (1995). Dictionnaire historique de l'orthographe française. Paris, France : Larousse.

Champoux, M. (2015). Portrait des difficultés du secondaire relativement à l'orthographe des formes homophones (Mémoire de maîtrise). Université de Montréal, Montréal, Canada. Repéré à https://papyrus.bib.umontreal.ca/xmlui/handle/1866/13985

Cogis, D. (2005). Pour enseigner et apprendre l'orthographe. Paris, Franc : Delagrave.

Cogis, D. et Brissaud, C. (2003). L'orthographe : une clé pour l'observation réfléchie de la langue? Repères, 28, 47-70.

David, R., Durand, M.-J., Giguère, M.-H., Lefrançois, P., Legendre, M.-F. et Plante, I. (2016). Étude des effets, sur les pratiques pédagogiques des enseignants et la compétence à écrire des élèves, d'un dispositif de formation à la nouvelle grammaire qui intègre des exemples de pratiques sur vidéo et permet un partage d'expertise (No. 2010-ER-137017). FRQSC, Programme actions concertées. Repéré à http://www.frqsc.gouv.qc.ca/partenariat/nos-resultats-de-recherche/histoire/etude- 
des-effets-sur-les-pratiques-pedagogiques-des-enseignants-et-la-competence-aecrire-des-eleves-d-un-dispositif-de-formation-a-la-nouvelle-grammaire-nouvellequi-integre-des-exemples-de-pratiques-sur-video-et-permet-un-partage-d-expertisgi7ja8p51481903588900

Desrosiers, H. et Tétrault, K. (2012). Les facteurs liés à la réussite aux épreuves obligatoires de français en sixième année du primaire : un tour d'horizon. Étude longitudinale du développement des enfants du Québec (ÉLDEQ 1998-2010)—De la naissance à 12 ans. Institut de la statistique du Québec, 7(1), 1-40.

Dionne, É. et Fleuret, C. (2016). L'analyse de données secondaires dans le cadre d'évaluation de programme : regard théorique et expérientiel. La Revue canadienne d'évaluation de programme, 31(2), (Fall/automne), 253-261. doi:10.3138/cjpe. 142.000

Duret, L. et Zecca, A. (2014). Peut-on enseigner efficacement les homophones grammaticaux en les étudiant séparément? Vers une nouvelle approche de l'enseignement des homophones grammaticaux (Mémoire professionnel de deuxième année). IUFM de Grenoble, Grenoble, France. Repéré à https://dumas.ccsd.cnrs.fr/dumas-00958890

Fisher, C. (1994). Les homophones dans l'enseignement de l'écrit, Dialangue, Bulletin de linguistique, 5, 9-16.

Gauvin, I. et Boivin, M.-C. (2013). Identifier le verbe : élaboration des connaissances par les élèves en classe. Revue des sciences de l'éducation, 39(3), 547570. http://dx.doi.org/10.7202/1026312ar.

Giguère M.-H. et Aldama R. (2019). Résoudre des problèmes liés à l'orthographe des homophones grammaticaux. Scolagram, 5(Pédagogie de la règle ou Didactique du truc ?), 1-25. Repéré à https://scolagram.ucergy.fr/index.php/content_page/item/280-resoudre-des-problemes-lies-a-1orthographe-des-homophones-grammaticaux

Goswami, U., Gombert, J.-E. et Barrera, L. F. d. (1998). Children's orthographic representations and linguistic transparency: Non-sense word reading in English, French, and Spanish. Applied Psycholinguistics, 19, 19-52.

Gourdet, P., Cogis, D. et Roubaud, M.-N. (2016). L'enseignement d'une notion-clé au primaire : le verbe. Dans S.-G. Chartrand (dir.), Mieux enseigner la grammaire (p. 147-174). Montréal, Canada : ERPI Éducation.

Jaffré , J.-P. (2006). Pourquoi distinguer les homophones? Langue française, 151, 25-40.

McNicoll, L. et Roy, G.-R. (1985). Les homophones: problèmes et solutions. Sherbrooke, Canada: Éditions Naaman.

McNicoll, L., Roy, G.-R. et Université de Sherbrooke. (1984). Programme de perfectionnement des maitres en français. Les homophones : diagnostics et remèdes. Sherbrooke, Canada : PPMF, Faculté d'éducation, Université de Sherbrooke.

Ministère de l'Éducation du Québec (MEQ). (1979). Programme d'études. Primaire. Français. Québec, Canada : Direction des programmes, Service du primaire.

Ministère de l'Éducation du Québec (MEQ). (2001). Programme de formation de l'école québécoise. Gouvernement du Québec. Repéré à http://www.education.gouv.qc.ca/references/publications/resultats-de-larecherche/detail/article/programme-de-formation-de-lecole-quebecoise-educationprescolaire-enseignement-primaire/ 
Ministère de l'Éducation, du Loisir et du Sport (MELS). (2009). Progression des apprentissages au primaire: Français, langue d'enseignement. Repéré à http://www.mels.gouv.qc.ca/sections/programmeFormation

Ministère de 1'Éducation, du Loisir et du Sport (MELS). (2011). Progression des apprentissages au secondaire. Français, langue d'enseignement. Repéré à http://www.mels.gouv.qc.ca/sections/programmeFormation

Mousty, P., et Alegria, J. (1999). L'acquisition de l'orthographe: données comparatives entre enfants normo-lecteurs et dyslexiques. Revue française de pédagogie, 7-22.

Nadeau, M. et Fisher, C. (2006). La grammaire nouvelle. La comprendre et l'enseigner. Boucherville, Canada : Gaétan Morin.

Nadeau, M. et Fisher, C. (2011). Les connaissances implicites et explicites en grammaire : quelle importance pour l'enseignement? Quelles conséquences ? Bellaterra Journal of Teaching and Learning Language and Litterature, 4(4), 1-31.

Nadeau, M. et Fisher, C. (2014). Expérimentation de pratiques innovantes, la dictée 0 faute et la phrase dictée du jour, et étude de leur impact sur la compétence orthographique des élèves en production de texte (No. 2010-ER-137018). FRQSC, Programme actions concertées. Repéré à http://www.frqsc.gouv.qc.ca/documents/11326/449040/PT_NadeauM_rapport+201 4_Dictée+impact+orthographique.pdf/f2307696-6f26-4ea0-965f-433a9fbe0a54

Office de la langue française du Québec. (2018). Banque de dépannage linguistique. «Qu'est-ce qu'un homophone lexical ? ». Repéré à http://bdl.oqlf.gouv.qc.ca/bdl/gabarit_bdl.asp?id=2645

Roy-Mercier, S. et Chartrand, S. (2016). L'enseignement du système de la conjugaison pour en favoriser l'apprentissage. Dans S.-G. Chartrand, S.-G. (dir.), Mieux enseigner la grammaire (p. 175-200). Montréal, Canada : ERPI Éducation.

Tallet, C. (2016a). L'enseignement des homophones grammaticaux à travers les manuels et les textes officiels. Le français aujourd 'hui, 3(194), 130. doi:10.3917/1fa.194.0071

Tallet, C. (2016b). Enseignement et apprentissage des homophones grammaticaux du CE2 à la $6^{\mathrm{e}}$ : bilan et perspectives. Pratiques [En ligne], 169-170. Repéré à http://pratiques.revues.org/3011. doi:10.4000/pratiques.3011 